\title{
Mercantilización de la cultura, del saber y de la información en la Sociedad del Conocimiento y problemas éticos derivados
}

\section{Mercantilization of culture, knowledge and information in the knowledge society and ethical problems arising}

\author{
Marta Bausá Arpón \\ marta.bausa.arpon@hotmail.com
}

\section{Resumen}

El objetivo de este trabajo es plantear algunos problemas éticos que pueden surgir del uso de las nuevas tecnologías de la comunicación, y que desafían en muchas ocasiones los valores tradicionales que se consideran establecidos en nuestra sociedad. Se pretende mostrar como las TIC han afectado de manera importante la evolución y desarrollo humano, y han modificado sustancialmente las relaciones interpersonales, especialmente en los jóvenes

\section{Palabras clave}

Sociedad del Conocimiento; Nuevas Tecnologías; Ética; Mercantilización de la cultura

\begin{abstract}
The purpose of this paper is to raise some ethical questions that may arise from the use of new communication technologies, and often challenging traditional values that are considered established in our society. It is intended to show how the New Technologies have significantly affected the evolution and human development, and have substantially changed interpersonal relationships, especially in young people
\end{abstract}

\section{Keywords}

Knowledge Society, New Technologies, Ethics, Mercantilization of culture

Recibido: 12/03/2016

Aceptado: 13/05/2016

DOI: http://dx.doi.org/10.5557/IIMEI7-N12-017041 
Descripción propuesta: BAUSÁ ARPÓN, Marta, 2016. Mercantilización de la cultura, del saber y de la información en la Sociedad del Conocimiento y problemas éticos derivados. Métodos de Información [en línea], 7(12), pp. 17-40.

\section{Introducción}

Las nuevas tecnologías han creado inmensas posibilidades que antes no estaban disponibles, también tiene aspectos negativos a los que hay que prestar atención y de los que se pretende hacer un pequeño esbozo en este trabajo.

Para empezar a abordar el tema objeto del presente trabajo, es necesario establecer las coordenadas sociales, económicas, políticas, técnicas y culturales que nos han llevado hasta la llamada Sociedad de la Información o Sociedad del Conocimiento.

Para ello se expondrá el paso de la Modernidad a la Postmodernidad como tránsito necesario hacia esa Sociedad de la Información y sus implicaciones culturales, políticas, sociales y económicas, y comprender así las transformaciones en el pensamiento de la sociedad. Se explicarán los conceptos de Sociedad de la Información y del Conocimiento y los riesgos que conllevan.

Ante los cambios impuestos por la alta tecnificación y por una sociedad cada vez más abierta y diversificada se hace indispensable observar en qué medida se ven alterados las tradiciones y los valores que dan perfil a las culturas. Lograr la convivencia de valores y principios humanitarios con las nuevas líneas de interacción planteadas por el posmodernismo es un reto al que debemos aspirar. Es por esto que este trabajo quiere abordar estos nuevos valores éticos que la Sociedad debe encontrar.

\section{La Postmodernidad}

El término postmodernidad es utilizado para designar generalmente a un amplio número de movimientos artísticos, culturales, literarios y filosóficos del siglo XX, que se extienden hasta hoy, definidos por su oposición o superación de las tendencias de la Edad Moderna. En sociología en cambio, los términos postmoderno y postmodernización se refieren al proceso cultural observado en muchos países en las últimas dos décadas, identificado a 
principios de los 70. Esta otra acepción de la palabra se explica bajo el término postmaterialismo.

El vertiginoso avance en la industria, la ciencia y la tecnología se ha constituido en el marco de profundas transformaciones socio-culturales que han caracterizado a las sociedades de finales del siglo XX. Hoy, por la mediación de las telecomunicaciones y de los medios masivos de comunicación, el ser humano tecnologizado interactúa en un contexto de interdependencia y globalidad. Esta tendencia, generada en los años más recientes, ha dado paso a la construcción de una nueva filosofía que define a la realidad como interpretación sustentada en lo diverso y lo plural (Anaya Ávila).

\subsection{Paso de la modernidad a la postmodernidad}

La posmodernidad es el enfoque que rompe con los criterios absolutistas y que produce novedosas concepciones sobre la multiplicidad de facetas que convergen alrededor de la realidad. Esta concepción de vida ha impactado sensiblemente el orden social y ha propiciado pautas de apertura democrática que, sin duda, pueden dar lugar a una revolución en el ámbito socio-cultural y político.

Tal y como explica Rifkin (2000), el inicio de la modernidad, se sitúa con el inicio de la época contemporánea, es decir desde la Ilustración francesa en siglo XVIII hasta finalizada la II Guerra Mundial, y significó el auge del pensamiento racional, el materialismo, el cientifismo, el progreso, la superación, la crítica, la vanguardia, la naturaleza, la realidad social como objeto de conocimiento objetivo, y las ideologías frente a la Teología del medievo, hubo una transición del concepto medieval de percepción por el moderno concepto de conocimiento objetivo.

Las diferentes corrientes del movimiento postmoderno aparecieron, como ya se ha dicho, durante la segunda mitad del siglo XX. Aunque se aplica a corrientes muy diversas, todas ellas comparten la idea de que el proyecto modernista fracasó en su intento de renovación radical de las formas tradicionales del arte y la cultura, el pensamiento y la vida social. Son muchos los autores que han tratado este tema: Jürgen Habermas, Jean-François Lyotard, Giannina Braschi, Andreas Huyssen, Gianni Vattimo, Baudraillard. 
Según Sandra Carli, el debate modernidad-posmodernidad surgió como respuesta a una época donde la mutación de referencias debilitó las verdades absolutas y proyectaron el futuro como una banalización de la historia. Lineal y extendido hacia un futuro determinado, la modernidad prevaleció la visión de un presente estable con miras hacia un progreso enmarcado por el desarrollo de las sociedades industriales.

Pero esta visión, a su vez, se fue distorsionando a medida que los avances tecnológicos comenzaron a generar cambios drásticos en las sociedades. Determinados, en parte, por la expansión del capital financiero, la transnacionalización de las economías, las transformaciones culturales y la creciente conectividad, los cambios fueron acelerándose dejando atrás a las instituciones y todas las estructuras pesadas que se oponían a una adaptación acorde con las nuevas lógicas posmodernas. Por tanto, la modernidad fue dando paso a contextos cada vez más complejos y caóticos, donde la velocidad de los cambios desequilibró el presente de los individuos para proyectarlos a un sin número de futuros llenos de incertidumbre e inestabilidad.

Según esta misma autora las discusiones filosóficas posmodernas centraron sus argumentos en tres tesis principales:

1. El fin de los grandes relatos e ideologías;

2. La relatividad del conocimiento, del estatuto de verdad y de los límites del saber;

3. La disolución de la idea del sujeto unitario y centrado.

\subsection{Un nuevo orden social}

Tal y cómo afirma María Del Pilar Anaya Ávila en su trabajo "De la modernidad hacia la posmodernidad en el uso de las nuevas tecnologías en la enseñanza superior", el posmodernismo está relacionado estrechamente con el capitalismo tardío, surge como paradigma que da cuenta del fracaso del Modernismo y que propicia, en consecuencia, un nuevo orden. En él, emerge un nuevo régimen social y económico, caracterizado por la gran tecnologización y el establecimiento de un mercado internacional concentrado en grandes bloques económicos. Paralelamente al ascenso financiero e industrial que identifica a esta etapa, en el ámbito de lo social, lo posmoderno se vincula al rechazo de los "grandes relatos" o "grandes proyectos" (metarrelatos, según François Lyotard) que proporcionaban algún tipo de 
cosmovisión. En el posmodernismo pierde sentido el planteamiento de visiones de la realidad totalizantes, coherentes e integradoras. Así, el pensamiento posmoderno se libera de la idea de unidad, y da paso al concepto de lo diverso. La diversidad y pluralidad dan significado a la nueva sociedad posmoderna y es el proceso de pluralización social el que conduce a una pluralización interna de los sujetos. Ellos, frente a una misma circunstancia, pueden comportarse de diferentes maneras, ya que se han transformado de sujetos aparentemente monoestructurales, a sujetos plurales.

La posmodernidad hace a un lado las viejas concepciones absolutistas y sustenta la necesidad de abordar la multiplicidad de facetas que confluyen en una realidad diversificada. Estas realidades son distintas y no pueden reducirse las unas a las otras. Luego entonces, la nueva personificación de la totalidad se construye a partir del enorme conglomerado de realidades que interactúan y superponen. La historia unitaria ya no existe y la realidad se vive como identidad fragmentada $\mathrm{y}$ dispersa. Es importante destacar, asimismo, que la nueva pluralidad, asociada a la capacidad de disentir sólo puede ser desarrollada en un régimen democrático que reconozca derechos fundamentales y derechos humanos.

\subsection{Otros enfoques. Puntos negros de la postmodernidad}

Continuando con el texto de Anaya Ávila, se puede afirmar que, si bien la posmodernidad propone nuevos cauces de apertura, es necesario reconocer que alrededor de sus bondades también se encubren enfoques plenos de pesimismo y desencanto. Así, el fin de los paradigmas totalizadores implica el fin -a nivel existencial- de marcos de referencia, lo cual conduce al caos y al irracionalismo. Ante el fracaso de las ideologías y la creciente brecha entre Norte y Sur, es más grande la desconfianza hacia el racionalismo sociopolítico y tecnológico. Además, la razón se ha convertido en instrumental, pues sólo sirve para la producción y el consumo.

$\mathrm{Al}$ ser desplazada la razón, priva el sentimiento y el individuo se convierte en un ser guiado en sus elecciones personales por el deseo. Este sujeto es escéptico, pesimista, hiperindividualista, hedonista y narcisista. La posmodernidad se erige hacia el campo de lo efímero, de lo inestable. El pasado se niega y las sociedades viven un presente perpetuo que arrasa 
tradiciones. En este contexto, se ha creado una crisis de valores que se desarrolla en diversos ámbitos de la vida social. En el caso específico de las creencias religiosas, éstas han dejado de ser sustento cultural y las grandes religiones institucionalizadas viven hoy una deserción masiva. Vivimos, por tanto, las consecuencias de una economía globalizada e interdependiente y del aceleradísimo flujo de información vertido a través de los medios masivos de comunicación. Ante este movimiento, se hace urgente rescatar el clima de respeto, tolerancia y pluralidad que promueve el posmodernismo y, a la vez, reorientar los valores que han padecido los embates de una sociedad compleja y caótica.

\section{Sociedad de la Información y Sociedad del Conocimiento}

Son muchos los autores que han tratado los conceptos de la Sociedad de la Información y de la Sociedad del Conocimiento y han definido sus características, y sus posibles diferencias. Con objeto de alcanzar una visión clara de estos dos conceptos se intentará hacer una revisión de ambos a través de los textos de varios autores.

\subsection{Sociedad de la Información}

El término 'Sociedad de la Información' ocupa un lugar estelar en la discusión actual en las ciencias sociales. Se trata de un concepto que aparentemente resume las transformaciones sociales que se están produciendo en la sociedad moderna y sirve para el análisis de estas transformaciones.

Es interesante, al respecto del concepto de Sociedad de la Información, el enfoque que Miguel Ángel Rendón Rojas hace en su artículo "Un análisis del concepto sociedad de la información desde el enfoque histórico". Propone identificar históricamente a la sociedad de la información de acuerdo con la forma en que la información se produce, se consume y se transmite. Rendón Rojas afirma que la Sociedad de la Información describe o intenta describir un tipo de sociedad que por un lado, ocupa un lugar histórico determinado y por otro, presenta características que la distinguen de otros tipos de sociedades históricas.

Se puede llegar a identificar históricamente a la sociedad de la información de acuerdo con la forma en que la información se produce, se consume y se transmite. De esta manera, y de acuerdo al criterio propuesto por Rendón Rojas, se pueden distinguir las siguientes etapas: 
1. Sociedad de tradición oral. En este tipo de sociedad la información fluye de manera oral, la información se transmite de generación en generación y su transformación es muy lenta.

2. Sociedad de elites privilegiadas con acceso a documentos manuscritos. Dentro de esta sociedad se tiene la posibilidad de registrar la información en documentos, aunque existe una serie de restricciones para poseerlos y asimilarlos. Se presenta una limitación para elaborar los documentos, debido a la producción manual de los soportes (papiro, pergamino, papel), así como al proceso de copiar los nuevos ejemplares en forma manuscrita. Al mismo tiempo, como consecuencia de que existe un número limitado de documentos, se presenta una restricción física en el acceso a la información registrada; aunque esa no es la principal, también existen una restricción cultural, porque son muy pocos los que saben leer, y una restricción de poder, porque sólo un pequeño grupo de elegidos es el que además de saber leer puede tener acceso al documento.

3. Sociedad del documento impreso (lectora). Gracias al invento de la imprenta y a la revolución cultural que esto motivó, se introdujo a la vida de la sociedad el documento impreso. En ese momento el acceso a la información se hace más general, lo que propicia que su circulación se haga más rápida. Este tipo de sociedad se caracteriza porque se reconoce como fuente de información principal el escrito impreso. Este último es el medio por excelencia empleado para guardar y transmitir información, aunque paulatinamente van apareciendo otros instrumentos para cumplir con esa función.

4. Sociedad de la Información. Finalmente, aparece un nuevo tipo de sociedad determinado por una serie de condiciones relacionadas entre sí y que según Randón Rojas motivan que la información aparezca como algo central e importante en la vida de la sociedad. Estas condiciones son las siguientes:

- Relaciones muy cambiantes y dinámicas en la estructura y funcionamiento de la sociedad. Se intensifica el ritmo de la movilidad y el cambio de todas las actividades y esferas de la vida social, lo cual exige a los individuos estar al día, actualizados en lo que acontece y evoluciona para no perderse en el mundo y poder actuar, tomando decisiones adecuadas en condiciones que pueden ser novedosas. 
- La globalización. Aparece una nueva configuración en la economía y sociedad mundial en la que se presentan una serie de procesos y problemas que abarcan a la mayor parte del planeta; se intensifican los niveles de interacción e interdependencia entre los Estados; y sucede que acontecimientos, decisiones y actividades de alguna región del mundo tienen repercusiones en los lugares más alejados del globo. (Dabat, 1999). De esta forma, se observa que el espacio en cierto sentido se rompe, lo que sucede en otra parte del mundo influye en las decisiones que se deben tomar, toda está conectado, por lo que la información fluye y actúa sin importar las barreras geográficas.

- La tecnología. La transformación y asimilación de la realidad se realiza con ayuda de una nueva herramienta cognitiva fruto de la unión de dos tipos de conocimiento: el técnico y el científico. El cómo hacer tiene a su servicio la racionalidad instrumental que crea objetos artificiales para controlar la realidad. Este factor, principalmente la conjunción del desarrollo computacional con el de las telecomunicaciones (y otros fenómenos tecnológicos que pueden aparecer ulteriormente) permite la rapidez del cambio, la globalización, la aceleración de la producción, flujo, transmisión y consumo de la información. Finalmente, podemos constatar que, gracias a la tecnología, no sólo el espacio sino también el tiempo se rompen.

- El mercado. La liberación, la privatización, la desregulación y la competencia como principios de la economía liberal (o neoliberal preferirán decir algunos) han provocado que las esferas productiva, de comercio, financiera, de servicios tengan alcances mundiales, sean versátiles y dinámicas y se conviertan en un juego económico que necesitan tener información a la mano lo más confiable, precisa y a tiempo posible, para que las jugadas que se realizan en ese juego impliquen menos riesgo y brinden la oportunidad de ganar. Debido a lo anterior, se crea una nueva esfera económica: el sector dedicado a la información. Es así que la información se convierte en un recurso estratégico, llega a ser un bien que posee un alto valor de uso, por lo que también adquiere un significativo valor de cambio, convirtiéndose 
consecuentemente en mercancía; pero una mercancía de tipo especial porque permite sobrevivir en el mercado (no sólo económico, sino en el neoliberalismo, donde todo es mercado, en el mercado político, científico, educativo, tecnológico, militar, etc.)

- El elevado nivel socio-económico. La preocupación inmediata de los individuos no es la subsistencia que ya está asegurada, sino que al tener un nivel de vida elevado, las necesidades aumentan y se hacen más complejas, por lo que se demanda un mayor progreso socio-económico, que se refleja en las exigencias al mercado y a la tecnología que responden motivando el flujo de la información, empezando el ciclo una vez más.

\subsection{Sociedad del Conocimiento}

La Sociedad del Conocimiento es un estadio de desarrollo social caracterizado por la capacidad de sus miembros para obtener, compartir y procesar cualquier información por medios telemáticos instantáneamente, desde cualquier lugar y en la forma que se prefiera. Hoy en día, la revolución de los medios digitales nos ha empujado a un nuevo orden económico y social que condiciona nuestro modo de ser en la sociedad. Es una transformación profunda, pero que se está desarrollando de forma muy desigual en distintos países.

La Sociedad de Conocimiento se caracteriza porque el conocimiento es el principal componente de cualquier actividad, ya sea económica, social o cultural. La información y el conocimiento son el principal recurso de toda actividad y también se constituyen en su producto. Se pueden enumerar los indicadores que caracterizan a esta Sociedad del Conocimiento de la siguiente manera:

- El conocimiento se produce mediante nuevas formas.

- El conocimiento es motor del crecimiento.

- Producción intensiva del conocimiento.

- Sistema autónomo de producción del conocimiento.

- Servicios basados en el conocimiento.

- Procesos educativos y formativos a lo largo de la vida.

- Ciudadanos trabajadores del conocimiento. 
- Uso intensivo de las TIC.

- Continúo cuestionamiento del conocimiento.

- Actuación basada en procesos de reflexión.

- Valoración continua de normas y reglas establecidas.

- Alta capacidad innovadora

- Procesos dinámicos transformadores permanentes.

- Conformada por subsistemas, redes, comunidades fuertemente vinculados.

- Asegura la producción, distribución y reproducción del conocimiento como proceso estratégico

- Sociedad cientificada.

- Sociedad con diferentes formas de organización y pensamiento social.

\subsection{La Sociedad del Conocimiento como sociedad postindustrial}

Tal y como acabamos de ver, la Sociedad del Conocimiento considera a las Tecnologías de Información y Comunicación (TIC) como un factor de cambio social. Es, por tanto una sociedad consciente del no-saber y de los riesgos de lo que esto implica.

Karsten Krüger en su trabajo "El concepto de 'Sociedad del Conocimiento"” hace un recorrido histórico sobre el origen de este concepto. Así, Krüger explica que la noción Sociedad de Conocimiento tiene sus orígenes en los años 60 cuando se analizaron los cambios en las sociedades industriales y se acuñó la noción de la sociedad post-industrial. Así, por ejemplo, el sociólogo Peter F. Drucker pronosticó la emergencia de una nueva capa social de trabajadores de conocimiento (Drucker 1959) y la tendencia hacia una sociedad de conocimiento (Drucker 1969). Este tipo de sociedad está caracterizada por una estructura económica y social, en la que el conocimiento ha substituido al trabajo, a las materias primas y al capital como fuente más importante de la productividad, crecimiento y desigualdades sociales.

Krüger continúa el recorrido histórico explicando el concepto de la sociedad post-industrial. Con este concepto se expresó la transición de una economía que produce productos a una economía basada en servicios y cuya estructura profesional está marcada por la preferencia a una clase de profesionales técnicamente cualificados. El conocimiento teórico se ha convertido, según 
este enfoque, en la fuente principal de innovación y el punto de partida de los programas políticos y sociales. Este tipo de sociedad está orientada hacia el progreso tecnológico y la evaluación de la tecnología y se caracteriza por la creación de una nueva tecnología intelectual como base de los procesos de decisión. En esta época, hasta el final de los años 70, el análisis de los cambios en la sociedad moderna resaltó tres aspectos:

- La expansión de las actividades de investigación, estatales y privadas, era la base principal de la cientificación de una serie de sectores industriales.

- Paralelamente a la expansión de los sectores de servicios, se incrementaron las actividades económicas basadas en el conocimiento. Con la importancia creciente del conocimiento teórico creció también el peso económico de las actividades basadas en el conocimiento.

- La estructura profesional estaba marcada por los trabajadores de conocimiento profesionalizado y con una cualificación académica: Bell (2001) estimó que alrededor de una cuarta parte de la población pertenecía a esta nueva clase de conocimiento, en la que se incluyó a los empleados con un diploma universitario o de una escuela superior, a los empleados y los funcionarios de altas categorías y a los empresarios.

Estos cambios produjeron una sociedad cientificada, academizada y centrada en los servicios diferenciada de la sociedad industrial que, a su vez, estaba caracterizada por el conocimiento experimental, el predominio de los sectores industriales, las actividades manuales y el conflicto entre capital y trabajo.

\section{Riesgos de la Sociedad del Conocimiento}

Cómo se ha podido ver en los epígrafes anteriores, son muchas las posibilidades que la Sociedad del Conocimiento brinda en la actualidad al conjunto de la Sociedad, pero no hay que perder de vista también los riesgos que conlleva. Algunos de los inconvenientes que están presentes en la Sociedad de la Información y del Conocimiento son:

- Se necesitan medios que no todo el mundo puede permitirse, creando desequilibrio social.

- Falta de comunicación entre personas si no hay un ordenador por medio.

- Deterioro de las relaciones humanas y pérdida de valores sociales. 
- Dependencia de las máquinas.

- Cada vez más pensamos menos y ejercitamos menos la mente y la imaginación.

- Falsa idea de que en Internet está toda la cultura.

En el siguiente epígrafe se esbozarán los riesgos de la Sociedad del Conocimiento que más preocupan y sobre los que han tratado numerosos autores.

\subsection{Paradoja de la Sociedad del Conocimiento: el crecimiento del no- conocimiento}

Continuando con el trabajo de Krüger, y como ya se ha dicho, se hace necesario también abordar las incertidumbres, las sombras, los dilemas y las paradojas de la Sociedad del Conocimiento. El sociólogo N. Stehr (2000) resalta, por ejemplo, la fragilidad de la sociedad del conocimiento moderno cuando subraya que los avances tecnológicos y científicos son una de las causas de la incertidumbre actual. Así, por ejemplo, los avances en las tecnologías de información y comunicación han aumentado la fragilidad de los mercados financieros y comerciales, lo cual obliga a las organizaciones a aumentar su flexibilidad para poder adaptarse a los cambios en los mercados. También el aumento del conocimiento científico y su amplia difusión causan más incertidumbre, fragilidad y contingencia (véase Stehr 1996). En este sentido se considera que el mayor conocimiento produce también más desconocimiento. Mientras los conocimientos aumentan con gran rapidez, el saber de lo que no sabemos aumenta con velocidad aún más vertiginosa. ( $\mathrm{H}$. D. Evers 2000). Por lo tanto, uno de los rasgos de la Sociedad del Conocimiento es el aumento de las zonas de incertidumbre, convirtiendo la ignorancia - entendida como el desconocimiento del no-conocimiento - en incertidumbre - entendido como el conocimiento del no-conocimiento (sé, que no sé).

\subsection{Riesgos de exclusión}

Por otro lado, es fácil percibir los nuevos riesgos de exclusión que existen en la Sociedad del Conocimiento. Los procesos socio-económicos cobran una nueva calidad porque el conocimiento se convierte en el factor de producción más importante. En este sentido, se está hablando de un nuevo modo de 
producción, dado que el capitalismo sigue siendo el principio dominante del sistema económico actual y no se oculta el riesgo de que aparezcan nuevas formas de exclusión social relacionadas con el conocimiento. Los riesgos de exclusión social en la sociedad del conocimiento están relacionados con el acceso a la información y al conocimiento, y con los efectos de la globalización socio-económica (Krüger).

Uno de los riesgos más apremiantes y discutidos en torno a la Sociedad de la Información es la llamada 'brecha digital', o lo que Krüger llama la 'división digital'. Este término hace referencia a las diferencias en el acceso a la información a través de las tecnologías de información y comunicación. En principio, los ordenadores y la Internet podrían facilitar la conexión de todas las personas a una red. En consecuencia, el acceso a la red y la capacidad de saber usarla es cada vez más importante para la participación en la vida social, económica y política. Por lo tanto, es importante la igualdad de oportunidad de poder acceder a la red y la capacitación de poder usar estos medios metódica y efectivamente.

\subsection{Mercantilización de cultura}

Tal y como señala Rifkin en su libro "La era del acceso" se ha producido un gran cambio en la sociedad, con el aumento y extensión de las tecnologías de la comunicación en la vida cotidiana, todas las formas de comunicación se están mercantilizando, y esta mercantilización ha llegado también al ámbito de la cultura, así el capitalismo no elabora sólo servicios sino también producciones culturales.

Siguiendo el análisis que hace María de Lourdes Ortiz Boza del texto de Rifkin en su artículo "Acceso y glocalidad: comunicación que se distancia", se expone como la nueva economía basada en el tercer sector, es decir el de servicios, esta nueva sociedad del siglo XXI, se caracteriza por el acceso. El acceso no solamente como la puerta de entrada a las Tecnologías de la Información y Comunicación Social y por lo tanto a la información, sino inclusive el acceso como estilo de vida, es decir, como una manera también excluyente de ver y relacionarse con los otros. En una sociedad cada vez más inaccesible, se da el fenómeno del acceso a la información, a las redes sociales, a la tecnología y, en fin, al nuevo orden mundial en materia de comunicaciones, que pareciera brindar a todos por igual ese acceso, cuando no es así. 
En "La nueva frontera del capitalismo", Rifkin desarrolla la entrada a la era del acceso, el momento en que los mercados dejan paso a las redes, la mercantilización de las relaciones humanas, la conversión de las ideas a producto mercadológico y el cómo todo lo humano se convierte en un servicio que se puede vender a través del acceso. Para comprender en qué consiste todo esto, Rifkin propone una serie de categorías que tienen que ver con el fenómeno de los conectados y los desconectados, los que tienen el acceso — limitado y excluyente— y los que no lo tienen, ni siquiera en lo más básico.

La autora (Ortiz Boza) habla de un sentido paradójico en esta era del acceso, pues la sociedad se encuentra en plena expansión mundial de la economía mediática y de servicios como en ninguna otra etapa de la historia humana, al brindar acceso a todos a través de la tecnología y las telecomunicaciones, especialmente Internet, al tiempo que los poseedores transnacionales de esas mismas tecnologías y medios crean sus propias comunidades de intereses y cercan al resto de la humanidad, con lo que niegan el acceso a su estilo de vida y lo limitan a aquellos que formen parte de esa élite poseedora del bien más preciado en este momento global: la información y la industria cultural del entretenimiento mediático. Según Rifkin, hoy estamos en el capitalismo cultural y la propiedad más relevante es precisamente la información y la cultura. Paradójicamente, dice Rifkin: "Mientras la quinta parte de la población mundial está migrando hacia el ciberespacio y hacia las relaciones de acceso, el resto de la humanidad está todavía atrapada en un mundo de escasez física".

Como podemos ver, la palabra clave para entender ese entorno en el cual se están moviendo hoy las comunidades, tanto desde las metrópolis tecnológicomediáticas, como las comunidades geográficas y culturales, es "acceso". De ahí que el autor hable de "La era del acceso"; quiénes están y quiénes no están teniendo acceso a las TICS y a los MMC (medios de comunicación de masas), ésa es la cuestión y aún más, dice Rifkin: "La brecha entre los poseedores y los desposeídos es ancha, pero la que existe entre conectados y desconectados es aún mayor. El mundo se desarrolla rápidamente en dos civilizaciones distintas: quienes viven dentro de las puertas electrónicas del ciberespacio y los que viven en el exterior". 
Otro autor que trata este tema es Mario Solano que en su artículo "La mercantilización del saber" expone también este concepto enmarcándolo dentro de la condición posmoderna. Para este autor, bajo las condiciones del capitalismo todos los productos adquieren la forma de mercancía, incluyendo a la capacidad de trabajo. Solano plantea que de manera reciente e íntimamente ligado a la expansión de las Nuevas Tecnologías Informáticas y Telemáticas, el saber mismo es producido y consumido como una mercancía y sugieren que esa producción es independizada crecientemente de la actividad de los propios seres humanos, en un proceso de externalización que, de estar ocurriendo, representaría una profundización de las tendencias reificantes del capitalismo señaladas por Marx.

En ese sentido, cabe destacar que un teórico de "la condición posmoderna", como lo es Lyotard, plantea no solo la actualidad de las categorías de la economía política en la llamada "era de la información", sino que en cierto sentido, le concede una gran validez histórica a estas, particularmente a la categoría de valor, cuando enuncia que el saber es transformado en las sociedades postindustriales en una mercancía que solo posee valor de cambio, y que por lo tanto pierde su valor de uso: "El saber es y será producido para ser vendido, y es y será consumido para ser valorado en un nueva producción: en los dos casos para ser cambiado. Deja de ser en sí mismo su propio fin, pierde su valor de uso" (Lyotard, 1998).

\section{Problemas éticos de la Sociedad el Conocimiento}

Por lo que hemos podido ver hasta ahora, son muchos los problemas y puntos negros que van asociados a la llamada Sociedad del Conocimiento. En este apartado se recogerán los problemas éticos derivados de la misma.

Tal y como apuntan $\mathrm{M}^{a}$ Ángeles San Martin y Manuel Sánchez en su artículo "Aspectos éticos de las nuevas tecnologías de la comunicación como agentes interactivos del desarrollo humano en la nueva era de la globalización", la globalización es un fenómeno de aperturas de culturas, de economías, de circulación de personas, ideas y nuevos conocimientos técnicos y científicos. Esta libre circulación de ideas, de formas de comunicar que ofrecen las tecnologías digitales, plantean diversos problemas y criterios éticos a tener en cuenta, ya que son millones las personas en el mundo que gozan del acceso a 
estas herramientas. Es importante dentro de este contexto global, tal y como señalan los autores, presentar recomendaciones para aquellos campos donde se requiere una sólida formación ética que permita elaborar, estructurar y difundir óptimamente, contenidos que respeten la libertad de expresión y de opinión, donde se respete la dignidad de la persona en toda su expresión, donde se tengan en cuenta los derechos de los menores y que al propio tiempo, sirva como rol social y educativo. Las nuevas generaciones están cada vez más familiarizadas y más en contacto con las llamadas nuevas tecnologías digitales, pero ¿están capacitados para hacer un uso correcto de ellas? ¿La educación que han recibido es la congruente con lo que circula por las redes? ¿Se plantean las personas si aquello que publican es éticamente correcto y apto para todos los navegantes? El punto principal es, si existe una formación ética suficientemente sólida como para discernir e interpretar lo que está delante de nuestros ojos.

Los conflictos éticos, por el uso inapropiado de las TIC, han estado presentes desde inicios de los años 70. Entre los aspectos negativos que destacan los autores se pueden señalar: la influencia que las TIC opera en los jóvenes y su nueva forma de enfocar los estudios, y que está variando su manera de entender la vida ya que las TIC no les ayuda a reflexionar, a cuestionar y analizar los conceptos importantes, sino a buscar soluciones rápidas; y la dependencia de las personas de las TIC en el mundo laboral y como afectan a su relación con el trabajo y la familia.

Para estos autores, los jóvenes son los más vulnerables a la globalización y a las Nuevas Tecnologías ya que son ellos los que actualmente crecen cada vez más próximos a los desarrollos tecnológicos y se ven más afectados por los efectos de la globalización, viven en contacto directo con el mundo multimediático y son una generación que el uso de las tecnologías les permite una interactividad y proximidad nunca antes conocida. La familiarización con las nuevas tecnologías con los jóvenes ha llegado a tal punto, que el lenguaje cibernético ha cambiado la forma de construir y concebir nuestra realidad.

Así, un aspecto que se ha detectado está siendo afectado por el uso continuo de las nuevas tecnologías es la disminución de la capacidad de la imaginación. Las nuevas tecnologías lo dan todo hecho. Ya no se inventa, lo que ahora se hace es planificar la búsqueda de información. Esto no tiene por qué ser malo, 
sino que debería ser un complemento y nunca una sustitución Otro aspecto a tener en cuenta es la expresión oral. Los niños y jóvenes están cada vez más acostumbrados a comunicarse a través de sms por los móviles o por correos electrónicos en internet. Su lenguaje es reducido, abreviado que unido a la falta de vocabulario por la escasa lectura, hace que la comunicación interpersonal y cara a cara, sea más difícil y menos expresiva, por lo que a los jóvenes les cuesta más comunicarse, especialmente con los adultos, con una clara exposición de ideas y argumentos.

Sin embargo, no sería correcto mantener una postura negativa frente al uso de las Nuevas Tecnologías, así los autores afirman: "todo lo expuesto hasta ahora no significa que las nuevas tecnologías sean un peligro o deban infundir miedo. Esto es una nueva cultura y una nueva forma de estar en el mundo. Los roles de la nueva sociedad, de los jóvenes, se encuentran en proceso de ajustes y de reconfiguraciones."

Uno de los problemas éticos relacionados con las Nuevas Tecnologías que más preocupan es la privacidad y la seguridad de las personas. Evidentemente, aquí entra una importante cuestión ética: ¿cómo se puede controlar lo que circula por la web? ¿Existe el respeto a la privacidad, entre otros, en un campo tan globalizado como son las redes sociales? La intimidad de las personas se ve muchas veces vulnerada por las grandes posibilidades de acceder a datos privados de forma fácil y rápida por expertos, y a veces no tan expertos en obtención de datos. El anonimato que provee Internet permite enviar contenidos emocionales que pueden resultar graves y ofensivos. El problema ético consiste no solo en defender la privacidad, sino en concienciar a las personas que la libertad de expresión no significa libertad para violar los derechos de otras personas. Debemos por lo tanto entender y aceptar todas las ventajas de las nuevas tecnologías, pero también tratar de buscar y orientar hacia unas nuevas formas de utilización de las mismas, donde las personas puedan verse beneficiadas y no perjudicadas por la actuación y el mal uso de personas sin escrúpulos o carentes de una responsabilidad ética.

Otros autores han tratado este mismo tema, así Linares Salgado en "Problemas éticos en torno a la sociedad del conocimiento" plantea varios de esos problemas: 
- El conocimiento como bien público vs. privatización del conocimiento. Un indicador positivo para la construcción de la sociedad del conocimiento sería la preservación de investigación básica que no esté subordinada directamente a fines pragmáticos. El segundo indicador debería ser la protección legal de conocimiento social de conocimiento de dominio público para evitar su privatización, así como la revisión de patentes relativas a información genética y seres vivos

- El desarrollo cognitivo equitativo, base del desarrollo social. Una Sociedad del Conocimiento no es aquella que sólo intercambia y comercia mundialmente con la información, sino aquella que es capaz de incrementar la generación de nuevos conocimientos e integrarlos con los anteriores, para lo cual pone a disposición de un mayor número de individuos redes de información de acceso abierto. Ello implica que el conocimiento sea un bien de dominio público. La Sociedad del Conocimiento se debería diferenciar de los restantes modelos sociales que ha habido en la historia por su capacidad para enfrentar los problemas mundiales mediante la colaboración científica, el intercambio libre del conocimiento, la deliberación plural y pública y la cooperación internacional.

- Límites y problemas de la libertad de expresión. Uno de los problemas éticos cruciales y más controvertidos en la sociedad del conocimiento, que emerge desde la sociedad de la información, es la necesidad o viabilidad de fijar límites (ética y jurídicamente) a la libertad de expresión y circulación de las ideas e informaciones. La libertad de expresión supone no sólo la libertad de opinar y publicar, sino también el libre acceso a la información y la libre circulación de datos e ideas. Por otro lado, otros dos fenómenos que se han exacerbado en la sociedad de la información se convierten en temas éticos de primer orden: la disolución de fronteras entre lo privado y lo público, y la capacidad de los Estados para vigilar muy de cerca las actividades y las opiniones, los gustos y las ideas de su población. La Sociedad del Conocimiento puede convertirse en una sociedad de la vigilancia mundial al estilo de la novela 1984 de Orwel, o bien hacer del ciberespacio un mundo inseguro en el que cualquiera puede sufrir daños a su patrimonio, ser víctima del fraude digital, padecer el robo de 
su identidad y quizá ser inculpado falsamente de un crimen. ¿En qué debe consistir el derecho a la privacidad en la sociedad del conocimiento? ¿Quién y cómo debe garantizarla seguridad informacional en la sociedad del conocimiento?

- La vulnerabilidad de la información y de la memoria. Un problema de no menor importancia en la Sociedad del Conocimiento es el de la vulnerabilidad de la información digital. Las bases de datos y los soportes electrónicos harán más vulnerables la información a pérdidas accidentales o destrucción intencionada. La mayor vulnerabilidad de la información y, por ende, mayor volatilidad del conocimiento, estriba en la aceleración del procesamiento de información La dirección que ha tomado el desarrollo de las TIC podría generar otro fenómeno contradictorio. Por un lado, el acrecentamiento de la capacidad de cálculo, procesamiento de información y generación de conocimiento; por otro, la posible gradual pérdida de la memoria social y de la capacidad para sostener los vínculos ético-políticos más básicos. La automatización o instrumentalización de la memoria podría conllevar una mengua gradual del esfuerzo individual para memorizar. La memoria supone la integración de una experiencia compartida o significativa para una comunidad. Es decir, se refiere al fenómeno de la memoria social sobre hechos significativos de la historia de una nación o comunidad. En algunos casos, la reconstrucción o rescate de la memoria colectiva reviste un profundo significado ético y político, una premisa indispensable para el reclamo de hacer justicia a las víctimas del pasado. Sin embargo, esta instrumentalización u objetivación de la memoria ha convertido a ésta en algo demasiado inestable y vulnerable; el recuerdo se ha reducido al ámbito privado y subjetivo. El testimonio que no queda registrado prácticamente resulta invisible, casi inexistente. No todo recuerdo forma parte de la memoria social, no todo recuento de los hechos se convierte en testimonio.

\section{5. Ética en los profesionales de la información}

Parece interesante para finalizar este trabajo, un pequeño apunte sobre la ética que debe mantener los profesionales de la información ante la nueva Sociedad del Conocimiento y los retos que ésta plantea. 
Para Rodríguez Sánchez, Mesa Fleitas y Álvarez Solórzano, las entidades de información presentan características singulares que conllevan a los deberes morales del profesional en la actividad científico-investigativa, específicamente en el campo de la información científica, por ser este su contenido fundamental de trabajo. La actividad que precisa la generación de actividad científica implica un reto diario para el profesional de la información, debido al significado generado por la sociedad del conocimiento existente, que deben lidiar constantemente con el uso de las tecnologías y la satisfacción del usuario. Actualmente, no se concibe la generación, procesamiento y difusión de la información científica sin la tecnología, un aspecto muy positivo por las facilidades que esta brinda. Pero cabe preguntarse cuánto y hasta dónde se maneja la tecnología por los profesionales de información y por sus usuarios. La tecnología no representa un problema en cuestiones de ética, siempre que éticamente se pueda utilizar, y se precisa de un enfoque holístico y sinérgico que estreche estos dos conceptos.

Para estos autores, al analizar el desempeño moral del bibliotecario en la recolección, procesamiento, generación y difusión de información, se evidencia que intervienen diversos procesos que requieren actitudes pertinentes para efectuar esta actividad de manera perfecta. $Y$ señalan algunas de ellas:

- Se debe mantener una actitud disciplinada y organizada en el trabajo para evitar que la información recolectada no sea inadecuada.

- Se han de anular estereotipos existentes por la práctica de religiones diferentes por cuestiones raciales o diferencias socio-políticas.

- El personal debe identificar las necesidades de información en caso que el usuario no sepa expresarlas debidamente.

- Se ha de incentivar la duda científica que puede ser cubierta por el análisis de la información existente.

- Se ha de mantener una postura flexible a las críticas y aceptar éstas como procesos constructivos que mejorarían la cotidianidad de la labor bibliotecológica.

- Se debe establecer una familiarización entre el usuario y el centro.

- Se ha de proporcionar la comodidad de la expresión oral para solucionar el desconocimiento, sea para el manejo de la tecnología del centro o para la localización de la información. 
- El profesional de la información debe conservar una apariencia afable y netamente profesional.

- Se ha de evitar subestimar al usuario.

- Se ha de evitar la resistencia al cambio.

- Se debe mantener una superación constante.

- Se ha de tomar interés en el asunto de investigación planteada por el usuario, etcétera.

En estos autores predomina la importancia del usuario y del individuo frente a otros aspectos, explicando que el bibliotecario ha de mantener absoluta conciencia de que el usuario es su razón de existir dentro de la comunidad científica. Debe concebir al usuario como un sujeto que necesita satisfacer una necesidad de información que presenta una repercusión e impacto social y al cual se brinda información para apoyar sus procesos de toma de decisiones estratégicas. Por tanto, se puede plantear que del desarrollo de los deberes morales del bibliotecario depende el desarrollo de la actividad de un sujeto el que, a partir de su inteligencia desarrollará nuevos proyectos de investigación que se concretarán en un bien social, político y económico. Y así, continuando con este enfoque, la formación y educación de usuarios es otro de los elementos que urge de principios éticos en el bibliotecario moderno. Se parte de la idea de contribuir al desarrollo de actitudes, como es el caso de la búsqueda de información por diversos medios tecnológicos, como las bases de datos o la propia navegación en la red, así como con el manejo de un catálogo tradicional y en línea.

Se podría concluir diciendo, que el profesional de la información es un sujeto formado por parámetros morales individuales, que es afectado por estados anímicos y cambios políticos, sociales y económicos; desprenderse de ciertos apegos, estereotipos y tradiciones resulta difícil; sin embargo, esto puede ser manejable si la resistencia al cambio es muy baja. La capacidad de ser flexibles es muy importante para incorporar un código ético a la labor diaria del profesional de la información.

\section{Conclusiones}

Son muchas las posibilidades que la Sociedad del Conocimiento brinda en la actualidad al conjunto de la Sociedad, pero no hay que perder de vista también 
los riesgos que conlleva. Es necesario subrayar el hecho de que no se debe sobre valorar la información y la tecnología como instrumentos que conducirán automáticamente al desarrollo del ser humano. Los problemas propiamente humanos, los que se preguntan por el sentido del ser y de la existencia, del bien y del mal, de la verdad y de la belleza, de la libertad, de la responsabilidad, de la trascendencia, del sufrimiento y de la felicidad, no pueden ser resueltos por la ciencia, mucho menos por la información o la tecnología.

La Sociedad de la Información no es sinónimo de sociedad justa, democrática o más humana: "La democracia, la igualdad, la justicia y la verdad son valores que se recrean por seres humanos, no son algo estático y dados por un modelo y un instrumento" (Rendón Rojas, 2000). Por tanto, la existencia de los valores depende de la elección libre y consciente de los individuos, no son el resultado de la existencia de objetos tecnológicos.

A modo de conclusión, se plantean varias plantean varias preguntas al respecto de las cuestiones éticas derivadas del uso de las TICS que desde luego no tienen fácil respuesta y que merece la pena señalar recogidas por San Martin y Sánchez Cid: ¿Cómo podemos enfrentarnos a situaciones donde la publicación de cierto material puede causar graves daños y donde nadie tiene responsabilidad y por lo tanto no sufre castigo o sanción alguna? ¿Quién decide qué información sobre un personaje empresarial puede ser privada o pública? ¿Cómo se puede controlar el envío de datos personales sobre personas privadas a empresas con fines comerciales? ¿Cómo podemos controlar el material que publican nuestros jóvenes, el lenguaje que utilizan, las opiniones, los juicios de valor, los comentarios banales sobre temas importantes?" Y continúan añadiendo: "La concepción del bien y del mal parece que depende más del criterio personal que de los resultados de las acciones de las personas. Si la sociedad cree que "todo" debe estar a disposición de "todo" el mundo y que "todo" el mundo debe tener acceso a "todo" tipo de información, entonces ¿dónde estamos dejando nuestra responsabilidad y nuestra capacidad crítica y analítica como parte integrante de esta sociedad y de los medios de comunicación de masas, llámense internet? ¿A quién estamos transfiriendo esta responsabilidad? ¿Está quedando a merced del mejor postor?". Todas ellas son preguntas de gran calado y 
profundidad que la sociedad deberá responder si queremos construir un futuro mejor.

\section{Bibliografía}

ANAYA AVILA, María Del Pilar, 2015. De la modernidad hacia la posmodernidad en el uso de las nuevas tecnologías en la enseñanza superior. En: Monografias.com. [Consulta: 20 enero 2015]. Disponible en: http:/ / www.monografias.com/trabajos15/modernidad-

posmodernidad/modernidad-posmodernidad.shtml

ANDERSON, Perry, 2000. Los origenes de la posmodernidad. Anagrama. Madrid. ISBN 84-339-0591-0.

AROCENA, Felipe, 1991. La modernidady su desencanto, Vintén Editor, Montevideo. ISBN 9974-570-01-8.

BALLESTEROS, Jesús, 1984. Postmodernidad: decadencia o resistencia. Madrid, Tecnos, ( $4^{\mathrm{o}}$ ed. $)$

BALLESTEROS, Jesús, 2000. Postmodernidad y tercer milenio. Persona y derecho, $\mathrm{n}^{\circ}$ 43, pp. 15-32.

BAUDRILLARD, J., HABERMAS, J., SAID, E. [et al.], 2000. La posmodernidad. Kairós. ISBN 84-7245-154-2

DABAT, Alejandro. 1999. La globalización en perspectiva histórica. En: Muñoz García, Humberto y Roberto Rodríguez, ed. La sociedad mexicana frente al tercer milenio. Tomo I. México: UNAM, Coordinación de Humanidades; Ed. Miguel Angel Porrúa. p. 57-79.

FOSTER, H., [et al], 1985. La posmodernidad. Barcelona: Kairós.

HABERMAS, Jürgen. 1990. El discurso filósofico de la modernidad. En: El pensamiento posmetafísico. Madrid: Taurus. ISBN 84-306-1300-5

LINARES SALGADO, Jorge E., 2015. Problemas éticos en torno a la sociedad del conocimiento [en línea]. En: Academia.edu. [Consulta: 19 enero 2015]. Disponible en: 
http://www.academia.edu/592565/Problemas_\%C3\%A9ticos_de_la_sociedad_del _conocimiento

LÓPEZ LÓPEZ, Pedro; MORILLO CALERO, M ${ }^{\mathrm{a}}$ Jesús, 2003. Derecho a la información y democracia en el marco de la globalización neoliberal: bibliotecas, archivos y medios de comunicación de masas. Revista General de Información y Documentación, 13(2), 97-131. ISSN- 1132-1873. Disponible en: http:/ / revistas.ucm.es/index.php/RGID/article/view/RGID0303220097A

LÓPEZ LÓPEZ, Pedro, 2001. Derechos de información, medios de comunicación y democracia. Revista general de información y documentación, 11(2), 61-92. ISSN 11321873.

Disponible en: http:/ / revistas.ucm.es/index.php/RGID/article/view/RGID0101220061A

LYOTARD, Jean-François, 2006. La condición posmoderna: Informe sobre el saber Madrid: Catedra. ISBN 84-376-0466-4

ORTIZ BOZA, María de Lourdes, 2011. Acceso y glocalidad: comunicación que se distancia. Reseña de "La era del acceso. La revolución de la nueva economía" de Jeremy Rifkin. Contribuciones desde Coatepec [en línea] no. 21, julio-diciembre, pp. 141144. [Consulta: 18 enero 2015]. Disponible en:

http://www.redalyc.org/pdf/281/28122683009.pdf

RENDÓN ROJAS, Miguel Ángel, 2001. Un análisis del concepto sociedad de la información desde el enfoque histórico. Información, cultura y sociedad, nº 4, p. 9-21. ISSN 1851-1740. [Consulta: 19 enero 2015]. Disponible en: http:/ / revistascientificas.filo.uba.ar/index.php/ICS/article/view/1009/989

RODRÍGUEZ SÁNCHEZ, Yaniris; MESA FLEITAS, María Elena y ÁLVAREZ SOLÓRZANO, Ernestina, 2006. La ética del profesional de la información ante la nueva sociedad del conocimiento. Acimed 14(1). [Consulta: 18 enero 2015]. Disponible en: http://bvs.sld.cu/revistas/aci/vol14_1_06/aci12106.htm

SAN MARTÍN PASCAL, Ma Ángeles; SÁNCHEZ CID, Manuel, 2011. Aspectos éticos de las nuevas tecnologías de la comunicación como agentes interactivos del desarrollo humano en la nueva era de la globalización. En: Actas II Congreso Internacional Sociedad Digital: espacios para la interactividad y la inmersión, vol. 1, pp. 395407. ISBN 978-84-939077-5-4, 
SOCIEDAD DEL CONOCIMIENTO. SINED (Sistema Nacional de Educación a Distancia), 2015 [en línea. [Consulta: 22 enero 2015]. Disponible en: https://www.sined.mx/sined/sociedadconocimiento/

SOLANO SOLANO, Mario A., 2007. La mercantilización del saber. Tecnología en Marcha [en línea], 20(3). Disponible en: http://firgoa.usc.es/drupal/files/c87bbfe571cf-0b79.pdf

VATTIMO, G.; MARDONES, J.M.; URDANABIA I, [et al.], 1990. En torno a la posmodernidad. Barcelona: Anthropos. ISBN 84-7658-234-X 